\title{
The Integrality of Speech in Multimodal Interfaces
}

\author{
MICHAEL A. GRASSO, DAVID S. EBERT, and TIMOTHY W. FININ \\ University of Maryland Baltimore County
}

\begin{abstract}
A framework of complementary behavior has been proposed which maintains that directmanipulation and speech interfaces have reciprocal strengths and weaknesses. This suggests that user interface performance and acceptance may increase by adopting a multimodal approach that combines speech and direct manipulation. This effort examined the hypothesis that the speed, accuracy, and acceptance of multimodal speech and direct-manipulation interfaces will increase when the modalities match the perceptual structure of the input attributes. A software prototype that supported a typical biomedical data collection task was developed to test this hypothesis. A group of 20 clinical and veterinary pathologists evaluated the prototype in an experimental setting using repeated measures. The results of this experiment supported the hypothesis that the perceptual structure of an input task is an important consideration when designing a multimodal computer interface. Task completion time, the number of speech errors, and user acceptance improved when interface best matched the perceptual structure of the input attributes.
\end{abstract}

Categories and Subject Descriptors: H.1.2 [Models and Principles]: User/Machine Systems-human factors; H.5.2 [Information Interfaces and Presentation]: User Interfacesevaluation/methodology; input devices and strategies; interaction styles; H.5.3 [Information Interfaces and Presentation]: Group and Organization Interfaces-theory and models; J.3 [Computer Applications]: Life and Medical Sciences

General Terms: Design, Experimentation, Human Factors, Measurement, Performance, Theory

Additional Key Words and Phrases: Direct manipulation, input devices, integrality, medical informatics, multimodal, natural-language processing, pathology, perceptual structure, separability, speech recognition

\section{INTRODUCTION}

For many applications, the human-computer interface has become a limiting factor. One such limitation is the demand for intuitive interfaces for

This reseach was supported in part by grant 2R44RR07989-02A2 from the National Center for Research Resources.

Authors' address: Department of Computer Science and Electrical Engineering, University of Maryland Baltimore County, 1000 Hilltop Circle, Baltimore, MD 21250; email: grasso@cs.umbc.edu; http://www.cs.umbc.edu/ mikeg; ebert@cs.umbc.edu; finin@cs.umbc.edu. Permission to make digital/hard copy of part or all of this work for personal or classroom use is granted without fee provided that the copies are not made or distributed for profit or commercial advantage, the copyright notice, the title of the publication, and its date appear, and notice is given that copying is by permission of the ACM, Inc. To copy otherwise, to republish, to post on servers, or to redistribute to lists, requires prior specific permission and/or a fee.

(C) 1999 ACM 1073-0516/98/1200-0303 \$5.00 
nontechnical users, an important obstacle to the widespread acceptance of computer automation [Landau et al. 1989]. Another difficulty consists of hands-busy and eyes-busy restrictions, such as those found in the biomedical area during patient care or other data collection tasks. An approach that addresses both of these limitations is to develop interfaces using automated speech recognition. Speech is a natural form of communication that is pervasive, efficient, and can be used at a distance. However, widespread acceptance of speech as a computer interface has yet to occur.

This effort sought to cultivate the speech modality by evaluating it in a multimodal environment with direct manipulation. Preliminary work on this effort has already been published [Grasso et al. 1997]. The focus was to develop an empirically based model that can help predict the success of using speech in a multimodal interface [Cole et al. 1995]. The specific objective of this study was to apply the theory of perceptual structure to a multimodal interface using speech and mouse input. This was based on previous work with multimodal interfaces [Cohen 1992; Oviatt and Olsen 1994] and work that extended the theory of perceptual structure to unimodal interfaces [Jacob et al. 1994].

\section{MULTIMODAL INTERFACES}

The history of research in multimodal speech and direct-manipulation interfaces has led to the identification of two essential principles relevant to this research: the complementary framework between speech and direct manipulation, and contrastive functionality. Both principles are introduced along with general background information.

\subsection{Speech Interface}

Speech interfaces have a number of unique characteristics when compared to traditional modalities. The most significant is that speech is temporary. Once uttered, auditory information is no longer available. This can place extra memory burdens on the user and severely limit the ability to scan, review, and cross-reference information. Speech can be used at a distance, which makes it ideal for hands-busy and eyes-busy situations. It is omnidirectional and can communicate with multiple users, which has implications relating to privacy. Finally, more than other modalities, there is the possibility of anthropomorphism when using a speech interface. It has been documented that users tend to overestimate the capabilities of a system if a speech interface is used and that users are more tempted to treat the device as another person [Jones et al. 1990].

At the same time, speech recognition systems often carry technical limitations, such as speaker dependence, continuity, and vocabulary size. Speaker-dependent systems must be trained by each individual user, but typically have higher accuracy rates than speaker-independent systems which can recognize speech from any person. Continuous-speech systems recognize words spoken in a natural rhythm while isolated-word systems require a deliberate pause between each word. Although more desirable, 
continuous speech is harder to process, because of the difficulty in detecting word boundaries. Vocabulary size can vary anywhere from 20 words to more than 40,000 words. Large vocabularies cause difficulties in maintaining recognition accuracy, but small vocabularies can impose unwanted restrictions. A more thorough review can be found elsewhere [Peacocke and Graf 1990].

\subsection{Direct Manipulation}

Direct manipulation, made popular by the Apple Macintosh and Microsoft Windows graphical user interfaces, is based on the visual display of objects of interest, the selection by pointing, rapid and reversible actions, and continuous feedback [Shneiderman 1983]. The display in a direct-manipulation interface should indicate a complete image of the application's environment, including its current state, what errors have occurred, and what actions are appropriate. A virtual representation of reality is created, which can be manipulated by the user through physical actions like pointing, clicking, dragging, and sliding.

While this approach has several advantages, arguments have been made that direct manipulation is inadequate for supporting fundamental transactions in applications such as word processing, CAD, and database queries. These comments were made in reference to the limited means of object identification and how the nondeclarative aspects of direct manipulation can result in an interface that is too low level [Buxton 1993; Cohen and Oviatt 1994]. Shneiderman [1993] points to ambiguity in the meanings of icons and limitations in screen display space as additional problems.

\subsection{Complementary Framework}

It has been suggested that direct-manipulation and speech recognition interfaces have complementary strengths and weaknesses that could be leveraged in multimodal user interfaces [Cohen 1992]. By combining the two modalities, the strengths of one could be used to offset the weaknesses of the other. For simplicity, speech recognition was used to mean the identification of spoken words, not necessarily natural-language recognition, and direct manipulation focused on mouse input.

The complementary advantages of direct manipulation and speech recognition are summarized in Table I. Note that the advantages of one are the weaknesses of the other. For example, direct engagement provides an interactive environment that is thought to result in increased user acceptance and allows the computer to become transparent as users concentrate on their tasks [Shneiderman 1983]. However, the computer can only become totally transparent if the interface allows hands-free and eyes-free operation. Speech recognition interfaces provide this, but intuitive physical actions no longer drive the interface.

Considering these observations, a framework of complementary behavior proposed that direct-manipulation and speech interfaces have reciprocal strengths and weaknesses [Cohen and Oviatt 1994]. This implied that user 
Table I. Complementary Strengths of Direct Manipulation and Speech

\begin{tabular}{cc}
\hline Direct Manipulation & Speech Recognition \\
\hline Direct engagement & Hands/eyes free operation \\
Simple, intuitive actions & Complex actions possible \\
Consistent look and feel & Reference does not depend on location \\
No reference ambiguity & Multiple ways to refer to entities \\
\hline
\end{tabular}

Table II. Proposed Applications for Direct Manipulation and Speech

\begin{tabular}{cc}
\hline Direct Manipulation & Speech Recognition \\
\hline Visible References & Nonvisible References \\
Limited References & Multiple References \\
Simple Actions & Complex Actions \\
\hline
\end{tabular}

interface performance and acceptance could increase by adopting a multimodal approach combining the two. Several applications were identified where each modality would be beneficial as summarized in Table II. Direct-manipulation interfaces were believed to be best used for simple actions with a limited number of visible references, while speech would be better at specifying more complex actions when references are numerous and not visible.

\subsection{Contrastive Functionality}

Oviatt and Olsen [1994] examined how people might combine input from different devices in a multimodal computer interface. They used a simulated service transaction system with verbal, temporal, and computational input tasks using both structured and unstructured interactions. Participants were free to use handwriting, speech, or both during testing.

This study evaluated user preferences in modality integration using spoken and written input. Among the findings, it was noted that simultaneous input with both pen and voice was rare and that digits, proper names, and structured interactions were more likely written.

The most significant factor in predicting the use of integrated multimodal speech and handwriting was what they called contrastive functionality. Here, the two modalities were used in different ways to designate a shift in context or functionality. Input patterns observed were original versus corrected input, data versus command, and digits versus text. For example, one modality was used for entering original input, while the other was reserved for corrections.

While this study identified user preferences, a followup study explored possible performance advantages [Oviatt 1996]. It was reported that multimodal speech and handwriting interfaces decreased task completion time and decreased the number of errors. 


\section{THEORY OF PERCEPTUAL STRUCTURE}

Along with important principles of multimodal interfaces, the work we present is based on an extension of the theory of perceptual structure [Garner 1974]. Perception is a cognitive process that occurs in the head, somewhere between the observable stimulus and the response. This response is not just a simple representation of a stimulus, because perception consists of various kinds of cognitive processing with distinct costs. Pomerantz and Lockhead [1991] built upon Garner's work to show that by understanding and capitalizing on the underlying structure of an observable stimulus, a perceptual system could reduce these processing costs.

Structures that abound in the real world are used by people to perceive and process information. Structures are defined as the way the constituent parts are arranged to give something its distinctive nature and often involve redundancy. Relying on this phenomenon has led to increased efficiency in various activities. For example, a crude method for weather forecasting is that the weather today is a good predictor of the weather tomorrow. An instruction cache can increase computer performance because the address of the last memory fetch is a good predictor of the address of the next fetch. Software engineers use metrics from previous projects to predict the outcome of future efforts.

While the concept of structure has a dimensional connotation, Pomerantz and Lockhead [1991] state that structure is not limited to shape or other physical stimuli, but is an abstract property that transcends any particular stimulus. Under this viewpoint, information and structure are essentially the same in that they are the property of a stimulus that is perceived and processed. This allowed us to apply the concept of structure to a set of attributes that are more abstract in nature, i.e., the collection of histopathology observations.

\subsection{Integrality of Stimulus Dimensions}

Garner documented that the dimensions of a structure can be characterized as integral or separable, and that this relationship may affect performance under certain conditions [Garner 1974; Shepard 1991]. The dimensions of a structure are integral if they cannot be attended to individually, one at a time; otherwise, they are separable.

Whether two dimensions are integral or separable can be determined by similarity scaling. In this process, similarity between two stimuli is measured as a distance. Subjects are asked to compare pairs of stimuli and indicate how alike they are. For example, consider the three stimuli A, B, and $\mathrm{C}$. Stimuli $\mathrm{A}$ and $\mathrm{B}$ are in dimension $\mathrm{X}$ (they differ based on some characteristic of X). Similarly, stimuli A and $\mathrm{C}$ are in the $\mathrm{Y}$ dimension. Given the values of $d_{x}$ and $d_{y}$, which each differ in one dimension, the value of $d_{x y}$ can be computed.

The distance between $\mathrm{C}$ and $\mathrm{B}$, which are in different dimensions, can be measured in two ways, as diagrammed in Figure 1. The city-block or 


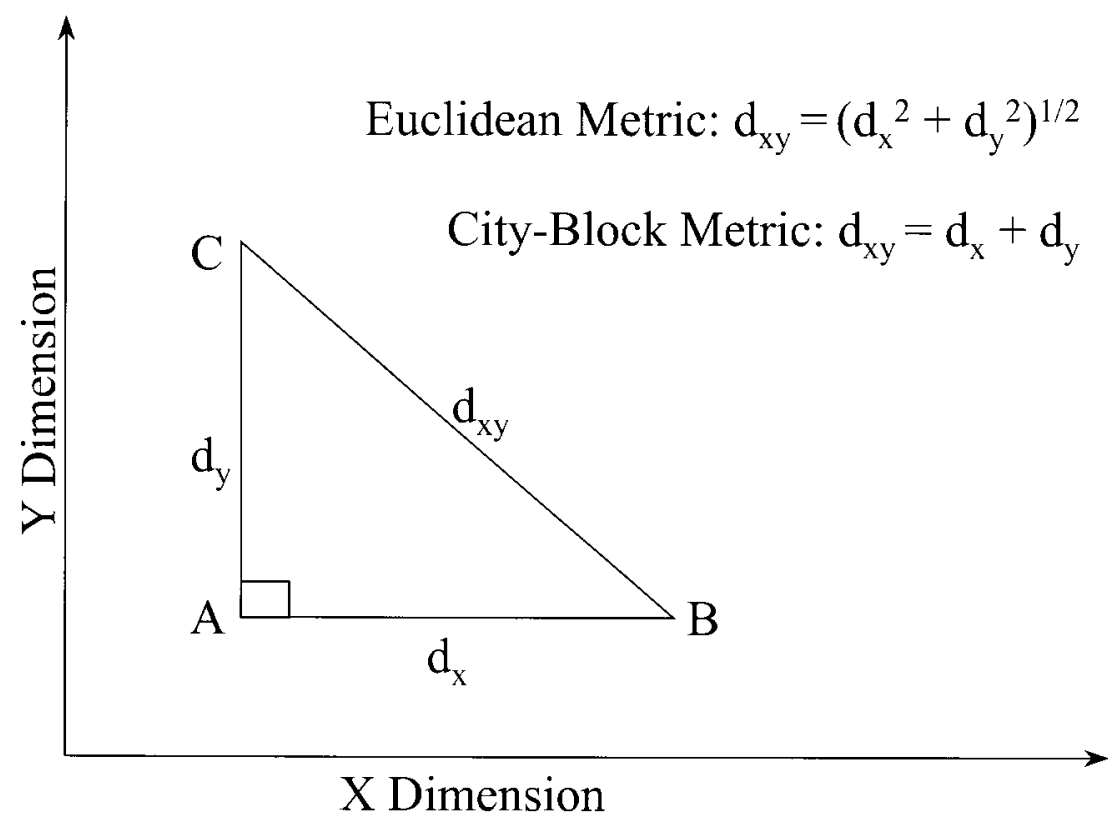

Fig. 1. Euclidean versus city-block metrics.

Manhattan distance is calculated by following the sides of the right triangle so that $\mathrm{d}_{\mathrm{xy}}=\mathrm{d}_{\mathrm{x}}+\mathrm{d}_{\mathrm{y}}$. The Euclidean distance follows the Pythagorean relation so that $d_{x y}=\left(d_{x}^{2}+d_{y}^{2}\right)^{1 / 2}$. This value is then compared to the value between $\mathrm{C}$ and $\mathrm{B}$ given by the subjects. If the given value for $\mathrm{d}_{\mathrm{xy}}$ is closer to the Euclidean distance, the two dimensions are integral. If it is closer to the city-block distance, the dimensions are separable.

\subsection{Integrality of Unimodal Interfaces}

Considering these principles, Jacob et al. [1994] tested the hypothesis that performance improves when the perceptual structure of the task matches the control structure of the input device. The concept of integral and separable was extended to interactive tasks by noting that the attributes of an input task correspond to the dimensions of an observable stimulus. In addition, certain input attributes would be integral if they follow the Euclidean metric, and separable if they follow the city-block metric.

Each input task involved one multidimensional input device, either a two-dimensional mouse or a three-dimensional tracker. Two graphical input tasks, each with three inputs, were evaluated: one where the inputs were integral ( $x$ location, $y$ location, and size) and the other where the inputs were separable (x location, y location, and color).

Common sense might say that a three-dimensional tracker is a logical superset of a two-dimensional mouse and therefore always as good and sometimes better than a mouse. Instead, the results showed that the 
tracker performed better when the three inputs were perceptually integral, while the mouse performed better when the three inputs were separable.

\subsection{Application of Perceptual Structure to Multimodal Interfaces}

Previous work on multimodal interfaces reported that such interfaces should result in performance gains [Cohen 1992]. It was also reported that a multimodal approach is preferred when an input task contains a shift in context [Oviatt and Olsen 1994]. This shift in context suggests that the attributes of those tasks were perceptually separable.

In addition, the theory of perceptual structures, integral and separable, was extended with the hypothesis that the perceptual structure of an input task is essential to the performance of unimodal, multidimensional input devices on multidimensional tasks [Jacob et al. 1994]. Their finding that performance increased when a separable task used an input device with separable dimensions suggests that separable tasks should be entered with separate devices in a multimodal interface. Also, since performance increased when integral tasks were entered with an integral device suggests that a single device should be used to enter integral tasks in a multimodal interface.

Based on these results, a followup question was proposed to determine the effect of integral and separable input tasks on multimodal speech and direct-manipulation interfaces. Predicted results were that the speed, accuracy, and acceptance of multidimensional multimodal input would increase when the attributes of the task are perceived as separable, and for unimodal input would increase when the attributes are perceived as integral. Three null hypotheses were generated:

$\left(\mathrm{H} 1_{0}\right)$ : The integrality of input attributes has no effect on the speed of the user.

$\left(\mathrm{H} 2_{0}\right)$ : The integrality of input attributes has no effect on the accuracy of the user.

$\left(\mathrm{H} 3_{0}\right)$ : The integrality of input attributes has no effect on acceptance by the user.

In this experiment, the theory of perceptual structure was applied to a computer interface similar to that of Jacob et al. [1994]. One important difference was that Jacob used a single multidimensional device, while we used multiple one-dimensional devices. Note that we viewed selecting items with a mouse as a one-dimensional task, while Jacob viewed selecting an $\mathrm{X}$ and $\mathrm{Y}$ coordinate with a mouse as a two-dimensional task. The attributes of the input task corresponded to the dimensions of the perceptual space. The structure or redundancy in these dimensions reflected the correlation in the attributes. Those dimensions that were highly correlated were integral, and those that were not were separable. The input modality consisted of two devices: speech and mouse input. Those input tasks that used one of the devices were using the input modality in an integral way and those 
Table III. Input Device Perception Versus Modality

\begin{tabular}{ccc}
\hline Input Device & Perception & Modality \\
\hline Speech Only & Integral & Unimodal \\
Mouse Only & Integral & Unimodal \\
Speech and Mouse & Separable & Multimodal \\
\hline
\end{tabular}

input tasks that used both devices were using the input modality in a separable way. This is shown in Table III.

Histopathologic data collection in animal toxicology studies was chosen as the application domain for user testing. Applications in this area include several significant hands-busy and eyes-busy restrictions during microscopy, necropsy, and animal handling. It used a highly structured, specialized, and moderately sized vocabulary with an accepted medical nomenclature. These and other characteristics made it a prototypical data collection task, similar to those required in biomedical research and clinical trials, and therefore was a good candidate for a speech interface [Grasso 1995].

\section{METHODOLOGY}

\subsection{Independent Variables}

The two independent variables for the experiment were interface type and task order. The actual input task was to enter histopathologic observations consisting of three attributes: topographical site, qualifier, and morphology. The site is a location on a given organ such as the alveolus, which is a topographical site of the lung. The qualifier is used to identify the severity or extent of the morphology, such as mild or severe. The morphology describes a histopathological observation, such as inflammation or carcinoma. Note that the input task was limited to these three items. In normal histopathological observations, there may be multiple morphologies and qualifiers. These were omitted for this experiment. For example, consider the following observation of a lung tissue slide consisting of a site, qualifier, and morphology: alveolus, multifocal, granulosa cell tumor.

The three input attributes corresponded to three input dimensions: site, qualifier, and morphology. After considering pairs of input attributes, it was concluded that qualifier and morphology (QM relationship) were related by Euclidean distances and therefore integral. Conceptually, this makes sense, since the qualifier is used to describe the morphology, such as multifocal, granulosa cell tumor. The qualifier had little meaning taken by itself. The site and qualifier (SQ relationship) were related by city-block distances and therefore separable. Again, this makes sense, since the site identified what substructure in the organ a tissue sample was taken from, such as alveolus or epithelium. Similar to SQ, the site and morphology (SM relationship) were related by city-block distances and separable. Based on these relationships and the general research hypothesis, Table IV predicted 
Table IV. Predicted Optimal Modalities for Computer-Human Interface Improvements

\begin{tabular}{lccc}
\hline & Data Entry Task & Perception & Modality \\
\hline (SQ) & Enter Site and Qualifier & Separable & Multimodal \\
$($ SM $)$ & Enter Site and Morphology & Separable & Multimodal \\
$($ QM) & Enter Qualifier and Morphology & Integral & Unimodal \\
\hline
\end{tabular}

Table V. Possible Interfaces Combinations for the Software Prototype

\begin{tabular}{lccccccc}
\hline Modality & Site & Qual & Morph & SQ & SM & QM & Interface \\
\hline 1. Mouse & M & M & M & - & - & + & \\
2. Speech & S & S & S & - & - & + & \\
3. Both & M & S & S & + & + & + & Congruent \\
4. Both & S & M & M & + & + & + & \\
5. Both & S & S & M & - & + & - & Baseline \\
6. Both & M & M & S & - & + & - & \\
7. Both & S & M & S & + & - & - & \\
8. Both & M & S & M & + & - & - & \\
\hline
\end{tabular}

which modality would lead to performance, accuracy, and acceptability improvements in the computer interface.

The three input attributes (site, qualifier, morphology) and two modalities (speech, mouse) yielded a possible eight different user interface combinations for the software prototype as shown in Table V. Predicted interface improvements are shown for each pair of attributes (SQ, SM, QM) identified with a "+" or "-" for a predicted increase or decrease, respectively. The third alternative was selected as the congruent interface, because the choice of input devices was thought to best match the integrality of the attributes. The fifth alternative was the baseline interface, since the input devices least match the integrality of the attributes.

The third and fifth alternatives were selected over other equivalent ones, because they both required two speech inputs, which appeared adjacent to each other on the computer screen. This was done to minimize any bias related to the layout of information on the monitor.

It might have been useful to consider mouse-only and speech-only tasks (interface alternatives one and two). However, because of performance differences between mouse and speech input, any advantages due to perceptual structure could not be measured accurately.

The three input attributes involved reference identification, with no declarative, spatial, or computational data entry required. This included the organ sites, which may be construed as having a spatial connotation. However, most of the sites used were not spatial, such as the epithelium, a ubiquitous component of most organs. Sites were also selected from a list as opposed to identifying a physical location on an organ, and were identified beforehand with each slide. This should have minimized any bias toward either direct manipulation or speech.

There were some limitations in using the third and fifth alternatives. Note in Tables III and IV that both the input device and the input 
Table VI. Structure of Input Device and Input Attributes

\begin{tabular}{lccc}
\hline & Relationship & Device & Attributes \\
\hline Alternative 3 (Congruent) & SQ & Separable & Separable \\
& SM & Separable & Separable \\
Alternative 5 (Baseline) & QM & Integral & Integral \\
& SQ & Separable & Integral \\
& SM & Separable & Separable \\
& QM & Separable & Integral \\
\hline
\end{tabular}

attributes can be integral or separable. Table VI describes the interface alternatives in these terms. The congruent interface compares a separable device with separable attributes and an integral device with integral attributes. The baseline interface compares a separable device with integral attributes and a separable device with separable attributes. However, neither interface compares an integral device with separable attributes.

One other comment is that these specific user interface tasks were not meant to identify the optimal method for entering data. In fact, most pathologists would consider using two input devices to enter histopathology observations to be counterproductive. The goal of this effort was not to develop an optimal user interface for a specific task, but instead to discover something about the efficiency of multimodal interfaces.

\subsection{Dependent Variables}

The dependent variables for the experiment were speed, accuracy, and acceptance. The first two were quantitative measures, while the latter was subjective.

Speed and accuracy were recorded by both the experimenter and the software prototype. Speed was defined as the time it took a participant to complete each of the 12 data entry tasks and was recorded to the nearest millisecond. Three measures of accuracy were recorded: speech errors, mouse errors, and diagnosis errors. A speech error was counted when the prototype incorrectly recognized a spoken utterance by a participant. This was because the utterance was misunderstood by the prototype or was not a valid phrase from the vocabulary. Mouse errors were recorded when a participant accidentally selected an incorrect term from one of the lists displayed on the computer screen and later changed his or her mind. Diagnosis errors were identified as when the input did not match the most likely diagnosis for each tissue slide. The actual speed and number of errors were determined by analysis of diagnostic output from the prototype, recorded observations of the experimenter, and review of audio tapes recorded during the study.

User acceptance data was collected with a subjective questionnaire containing 13 bipolar adjective pairs that has been used in other humancomputer interaction studies [Casali et al. 1990; Dillon et al. 1995]. The adjectives are listed in Table VII. The questionnaire was given to each participant after testing was completed. An acceptability index (AI) was 
Table VII. Adjective Pairs used in the User Acceptance Survey

\begin{tabular}{llll}
\hline \multicolumn{2}{c}{ User Acceptance Survey Questions } & \\
\hline 1. Fast & Slow & 8. Comfortable & Uncomfortable \\
2. Accurate & Inaccurate & 9. Friendly & Unfriendly \\
3. Consistent & Inconsistent & 10. Facilitating & Distracting \\
4. Pleasing & Irritating & 11. Simple & Complicated \\
5. Dependable & Undependable & 12. Useful & Useless \\
6. Natural & Unnatural & 13. Acceptable & Unacceptable \\
7. Complete & Incomplete & & \\
\hline
\end{tabular}

defined as the mean of the scale responses, where the higher the value, the lower the user acceptance.

\subsection{Subjects}

Twenty subjects from among the biomedical community participated in this experiment as unpaid volunteers between January and February, 1997. Each participant reviewed 12 tissue slides, resulting in 240 tasks for which data were collected. The target population consisted of veterinary and clinical pathologists from the Baltimore-Washington area. Since the main objective was to evaluate different user interfaces, participants did not need a high level of expertise in animal toxicology studies, but only to be familiar with tissue types and reactions. Participants came from the University of Maryland Medical Center (Baltimore, MD), the Veteran Affairs Medical Center (Baltimore, MD), the Johns Hopkins Medical Institutions (Baltimore, MD), the Food and Drug Administration Center for Veterinary Medicine (Rockville, MD), and the Food and Drug Administration Center for Drug Evaluation and Research (Gaithersburg, MD). To increase the likelihood of participation, testing took place at the subjects' facilities.

The 20 participants were distributed demographically as follows, based on responses to the preexperiment questionnaire. The sample population consisted of professionals with doctoral degrees (D.V.M., Ph.D., or M.D.), ranging in age from 33 to 51 years old; 11 were male; 9 were female; 15 were from academic institutions; 13 were born in the United States; and 16 were native English speakers. The majority indicated they were comfortable using a computer and mouse, and only one had any significant speech recognition experience.

The subjects were randomly assigned to the experiment using a withingroup design. Half of the subjects were assigned to the congruent-interfacefirst, baseline-interface-second group and were asked to complete six data entry tasks using the congruent interface and then complete six tasks using the baseline interface. The other half of the subjects were assigned to the baseline-interface-first, congruent-interface-second group and completed the tasks in the reverse order. Also counterbalanced were the tissue slides examined. Two groups of six slides with roughly equivalent difficulty were randomly assigned to the participants. This resulted in four groups 
Table VIII. Subject Groupings for the Experiment

\begin{tabular}{lccccc}
\hline & \multicolumn{2}{c}{ First Task } & & \multicolumn{2}{c}{ Second Task } \\
\cline { 2 - 3 } \cline { 5 - 6 } & Interface & Slides & & Interface & Slides \\
\hline B1C2 & Baseline & $1-6$ & & Congruent & $7-12$ \\
B2C1 & Baseline & $7-12$ & & Congruent & $1-6$ \\
C1B2 & Congruent & $1-6$ & & Baseline & $7-12$ \\
C2B1 & Congruent & $7-12$ & & Baseline & $1-6$ \\
\hline
\end{tabular}

based on interface and slide order as shown in Table VIII. For example, subjects in group B1C2 used the baseline interface with slides 1 through 6 followed by the congruent interface with slides 7 through 12 .

\subsection{Materials}

A set of software tools was developed to simulate a typical biomedical data collection task in order to test the validity of this hypothesis. The prototype computer program was developed using Microsoft Windows 3.11 (Microsoft Corporation, Redmond, WA) and Borland $\mathrm{C}++4.51$ (Borland International, Inc., Scotts Valley, CA).

The PE500+ was used for speech recognition (Speech Systems, Inc., Boulder, CO). The hardware came on a half-sized, 16-bit ISA card along with head-mounted microphone and speaker, and accompanying software development tools. Software to drive the PE500+ was written in $\mathrm{C}++$ with the SPOT application programming interface. The Voice Match Tool Kit was used for grammar development. The environment supported speakerindependent, continuous recognition of large vocabularies, constrained by grammar rules. The vocabulary was based on the Pathology Code Table [NCTR 1985] and was derived from a previous effort establishing the feasibility of speech input for histopathologic data collection [Grasso and Grasso 1994]. Roughly 1,500 lines of code were written for the prototype.

The tissue slides for the experiment were provided by the National Center for Toxicological Research (Jefferson, AK). All the slides were from mouse tissue and stained with H\&E. Pictures were taken at high resolution with the original dimensions of 36 millimeters by 24 millimeters. Each slide was cropped to show the critical diagnosis and scanned at two resolutions: 570 by 300 and 800 by 600 . All scans were at 256 colors. The diagnoses for the 12 slides are shown in Table IX.

The software and speech recognition hardware were deployed on a portable PC-III computer with a 12.1-inch, $800 \times 600$ TFT color display, a PCI Pentium-200 motherboard, 32MB RAM, and 2.5GB disk drive (PC Portable Manufacturer, South El Monte, CA). This provided a platform that could accept ISA cards and was portable enough to take to the participants' facilities for testing.

The main task the software supported was to project images of tissue slides on a computer monitor while subjects entered histopathologic observations in the form of topographical sites, qualifiers, and morphologies. Normally, a pathologist would examine tissue slides with a microscope. 
Table IX. Tissue Slide Diagnoses

\begin{tabular}{lcl}
\hline & Slide & \multicolumn{1}{c}{ Diagnosis (Organ, Site, Qualifier, Morphology) } \\
\hline Group 1 & 1 & Ovary, Media, Focal, Giant Cell \\
& 2 & Ovary, Follicle, Focal, Luteoma \\
& 3 & Ovary, Media, Multifocal, Granulosa Cell Tumor \\
& 4 & Urinary Bladder, Wall, Diffuse, Squamous Cell Carcinoma \\
& 5 & Urinary Bladder, Epithelium, Focal, Transitional Cell Carcinoma \\
& 6 & Urinary Bladder, Transitional Epithelium, Focal, Hyperplasia \\
& 7 & Adrenal Gland, Medulla, Focal, Pheochromocytoma \\
Group 2 & 8 & Adrenal Gland, Cortex, Focal, Carcinoma \\
& 9 & Pituitary, Pars Distalis, Focal, Cyst \\
& 10 & Liver, Lobules, Diffuse, Vacuolization Cytoplasmic \\
& 11 & Liver, Parenchyma, Focal, Hemangiosarcoma \\
& 12 & Liver, Parenchyma, Focal, Hepatocelluar Carcinoma \\
\hline
\end{tabular}

Table X. Congruent Interface Transcript

\begin{tabular}{|c|c|c|c|c|}
\hline & Time & Device & Action & Comment \\
\hline \multirow[t]{4}{*}{ Task 1} & 0 & Mouse & Press button to begin test. & \\
\hline & 3 & Mouse & Click on "media" & \\
\hline & 7 & Speech & "Select marked giant cell" & \\
\hline & 14 & Mouse & Click on "press continue" button & \\
\hline \multirow[t]{4}{*}{ Task 2} & 20 & Mouse & Click on "follicle" & \\
\hline & 29 & Speech & "Select moderate hyperplasia" & Recognition error \\
\hline & 36 & Speech & "Select moderate hyperplasia" & \\
\hline & 42 & Mouse & "Select moderate hyperplasia" & \\
\hline \multirow[t]{3}{*}{ Task 3} & 44 & Mouse & Click on "media" & \\
\hline & 50 & Speech & "Select moderate inflammation" & \\
\hline & 57 & Mouse & Click on "press continue" button & \\
\hline \multirow[t]{3}{*}{ Task 4} & 61 & Mouse & Click on "wall" & \\
\hline & 65 & Speech & "Select marked squamous cell carcinoma" & \\
\hline & 71 & Mouse & Click on "press continue" button & \\
\hline \multirow[t]{3}{*}{ Task 5} & 74 & Mouse & Click on "epithelium" & \\
\hline & 81 & Speech & $\begin{array}{l}\text { "Select moderate transitional cell } \\
\text { carcinoma" }\end{array}$ & \\
\hline & 89 & Mouse & Click on "press continue" button & \\
\hline \multirow[t]{3}{*}{ Task 6} & 94 & Mouse & Click on "transitional epithelium" & \\
\hline & 96 & Speech & $\begin{array}{l}\text { "Select marked transitional cell } \\
\text { carcinoma" }\end{array}$ & \\
\hline & 104 & Mouse & Click on "press continue" button & \\
\hline
\end{tabular}

However, to minimize hands-busy or eyes-busy bias, no microscopy was involved. Instead, the software projected images of tissue slides on the computer monitor while participants entered observations in the form of topographical sites, qualifiers, and morphologies. While this might have contributed to increased diagnosis errors, the difference in relative error rates from both interfaces could still be measured. Participants were also allowed to review the slides and ask clarifying questions before the test.

The software provided prompts and directions to identify which modality was to be used for which inputs. No menus were used to control the system. Instead, buttons could be pressed to zoom the slide to show detail, adjust 
Table XI. Baseline Interface Transcript

\begin{tabular}{|c|c|c|c|c|}
\hline & Time & Device & Action & Comment \\
\hline \multirow[t]{5}{*}{ Task 1} & 0 & Mouse & Press button to begin test & \\
\hline & 15 & Mouse & Click on "medulla" & Incorrect action \\
\hline & 20 & Speech & "Select medulla mild" & \\
\hline & 21 & Mouse & Click on "pheochromocytoma" & \\
\hline & 27 & Mouse & "press continue" button & \\
\hline \multirow{4}{*}{ Task 2} & 35 & Speech & "Select cortex marked" & \\
\hline & 39 & Mouse & Click on "pheochromocytoma" & \\
\hline & 42 & Speech & "Select cortex marked" & \\
\hline & 51 & Mouse & Click on "press continue" button & \\
\hline \multirow[t]{3}{*}{ Task 3} & 70 & Speech & "Select pars distalis moderate" & \\
\hline & 76 & Mouse & Click on "granulosa cell tumor" & \\
\hline & 77 & Mouse & Click on "press continue" button & \\
\hline \multirow{3}{*}{ Task 4} & 82 & Speech & "Select lobules marked" & Recognition error \\
\hline & 88 & Mouse & Click on "vacuolization cytoplasmic" & \\
\hline & 89 & Mouse & Click on "press continue" button & \\
\hline \multirow[t]{4}{*}{ Task 5} & 97 & Speech & "Select parenchyma moderate" & Recognition error \\
\hline & 101 & Mouse & Click on "hemangiosarcoma" & \\
\hline & 103 & Speech & "Select parenchyma moderate" & \\
\hline & 109 & Mouse & Click on "press continue" button & \\
\hline \multirow[t]{4}{*}{ Task 6} & 114 & Speech & "Select parenchyma marked" & Recognition error \\
\hline & 118 & Mouse & Click on "hepatocellular carcinoma" & \\
\hline & 124 & Speech & Click on "press continue" button & \\
\hline & 128 & Mouse & Click on "press continue" button & \\
\hline
\end{tabular}

the microphone gain, or go to the next slide. To minimize bias, all command options and nomenclature terms were visible on the screen at all times. The user did not need to scroll to find additional terms.

A sample screen is shown in Figure 2. In this particular configuration, the user would select a site with a mouse click and enter the qualifier and morphology by speaking a single phrase, such as moderate, giant cell. The selected items would appear in the box above their respective lists on the screen. Note that the two speech terms were always entered together. If a term was not recognized by the system, both would have to be repeated. A transcript for the congruent and baseline interfaces for one of the subjects is given in Tables $\mathrm{X}$ and $\mathrm{XI}$.

\subsection{Procedure}

A within-groups experiment that was fully counterbalanced on input modality and slide order was performed. Each subject was tested individually in a laboratory setting at the participant's place of employment or study. Participants were first asked to fill out the preexperiment questionnaire to collect demographic information. The subjects were told that the objective of this study was to evaluate several user interfaces in the context of collecting histopathology data and was being used to fulfill requirements in the Ph.D. Program of the Computer Science and Electrical Engineering Department at the University of Maryland Baltimore County. They were told that a computer program would project images of tissue slides on a 


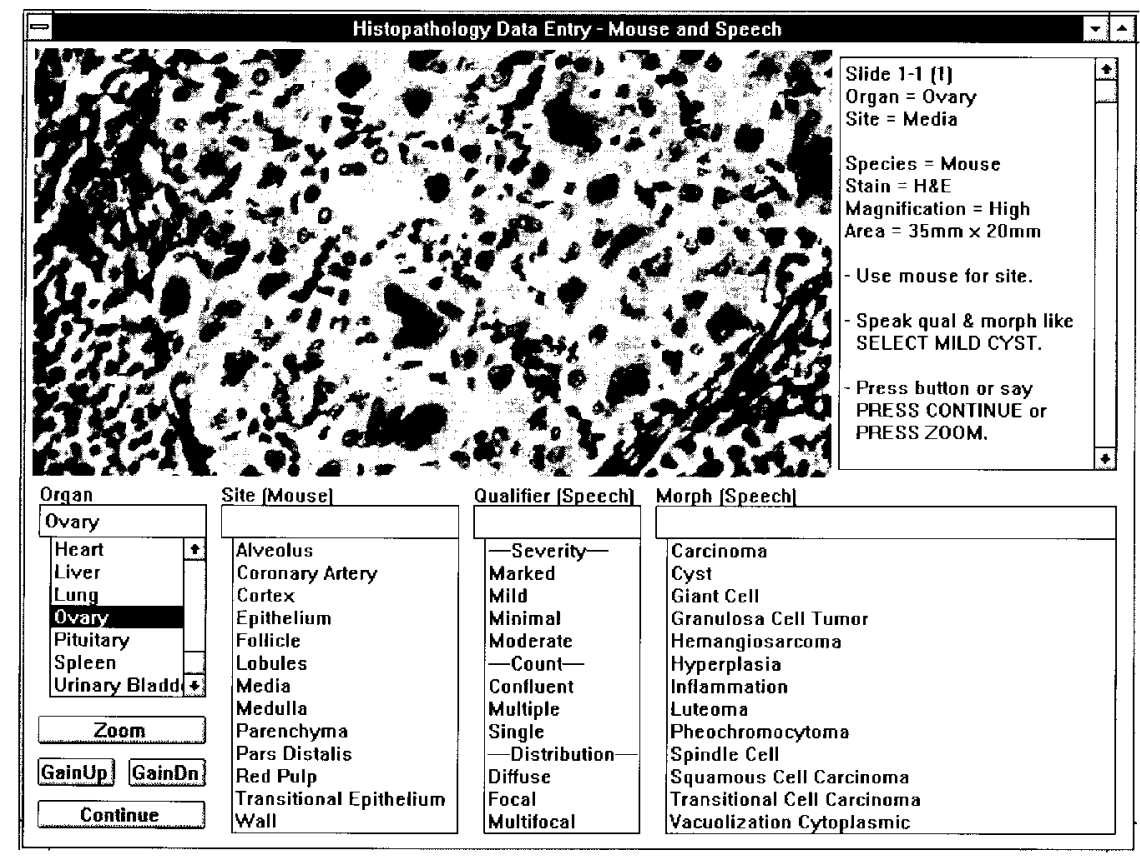

Fig. 2. Sample data entry screen.

computer monitor while they enter observations in the form of topographical sites, qualifiers, and morphologies.

After reviewing the stated objectives, each participant was seated in front of the computer and had the headset adjusted properly and comfortably, being careful to place the microphone directly in front of the mouth, about an inch away. Since the system came with a speaker-independent vocabulary provided with the PE500+ speech recognition engine, there was no need to enroll or train the speech recognizer. However, a training program was run to allow participants to practice speaking typical phrases in such a way that the speech recognizer could understand. The objective was to become familiar speaking these phrases with reasonable recognition accuracy. Participants were encouraged to speak as clearly and as normally as possible.

Next, each subject went through a training session with the actual test program to practice reading slides and entering observations. Participants were instructed that this was not a test and to feel free to ask the experimenter about any questions they might have.

The last step before the test was to review the two sets of tissue slides. The goal was to make sure participants were comfortable reading the slides. This was to ensure that the experiment was measuring the ability of subjects to enter data, not their ability to read slides. During the review, participants were encouraged to ask questions about possible diagnoses. 
Table XII. Experimental Procedure

\begin{tabular}{cl}
\hline Step & \multicolumn{1}{c}{ Task } \\
\hline 1 & Preexperiment questionnaire and instructions \\
2 & Speech training \\
3 & Application training \\
4 & Slide review \\
5 & Evaluation and quantitative data collection \\
6 & Postexperiment questionnaire and subjective data collection \\
\hline
\end{tabular}

For the actual test, participants entered two groups of six histopathologic observations in an order based on the group they were randomly assigned. They were encouraged to work at a normal pace that was comfortable for them and to ask questions before the test began. The user acceptance survey was administered as a postexperiment questionnaire. A summary of the experimental procedure can be found in Table XII.

\subsection{Results}

For each participant, speed was measured as the time to complete the six baseline interface tasks, the time to complete the six congruent interface tasks, and time improvement (baseline interface time - congruent interface time). The mean improvement for all subjects was 41.468 seconds. A $\mathrm{t}$-test on the time improvements was significant $(\mathrm{t}(19)=4.791, \mathrm{p}=$ 0.0001 , two-tailed). A comparison of mean task completion times is in Figure 3. For each subject, the six baseline and six congruent tasks are graphed.

A two-factor ANOVA with repeated measures was run as well. A $2 \times 4$ ANOVA was set up to compare the two interfaces with the four treatment groups. The sample variation comparing the baseline interface times to the congruent interface times was significant $(p=0.028)$. The ANOVA showed that the interaction between interface order and task order had no significant effect on the results $(\mathrm{p}=0.903)$.

Three types of user errors were recorded: speech recognition errors, mouse errors, and diagnosis errors. The baseline interface had a mean speech error rate of 5.35, and the congruent interface had mean of 3.40. The reduction in speech errors was significant (paired $t(19)=2.924, p$ $=0.009$, two-tailed). A comparison of mean speech error rates by task is shown in Figure 4. Similar to task completion times, a two-factor ANOVA with repeated measures was run for speech errors to show that the sample variation was significant $(\mathrm{p}=0.009)$ and that the interaction between interface order and task order had no significant effect on the results $(\mathrm{p}$ $=0.245)$

Mouse errors for the baseline interface had mean error rate of 0.35 , while the congruent interface had mean of 0.45 . These results were not significant (paired t t $(19)=0.346, p=0.733$, two-tailed). For diagnosis errors, 


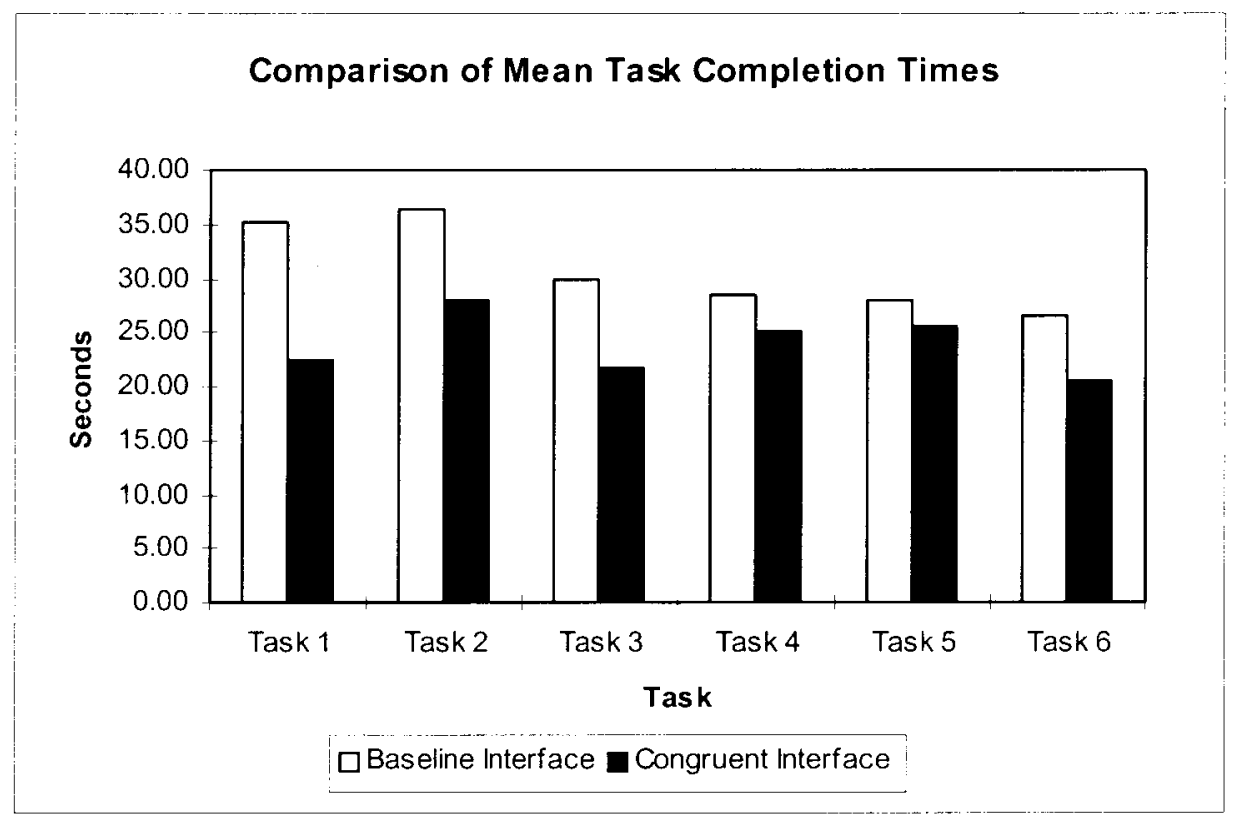

Fig. 3. Comparison of mean task completion times.

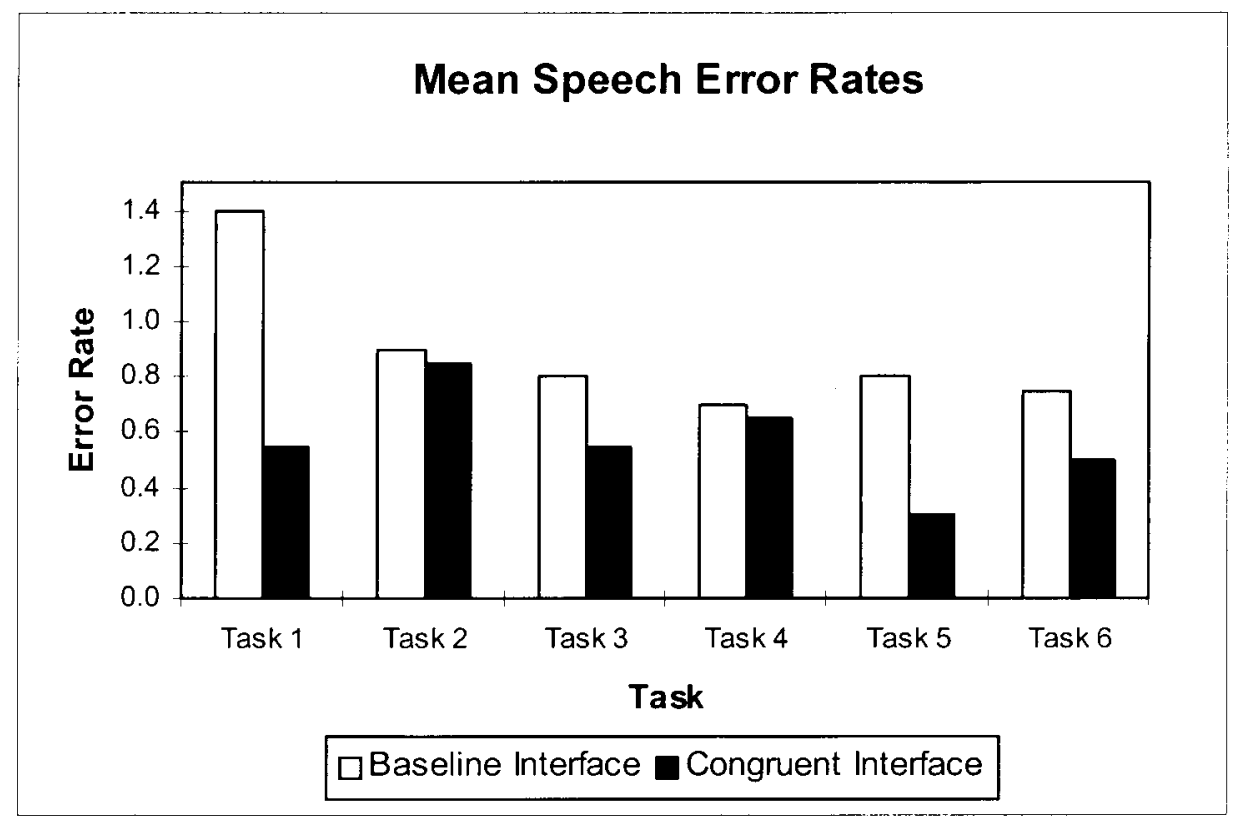

Fig. 4. Comparison of mean speech errors.

the baseline interface had mean error rate of 1.95 , and the congruent interface had mean of 1.90 . These were also not significant (paired $t(19)$ $=0.181, \mathrm{p}=0.858$, two-tailed). 


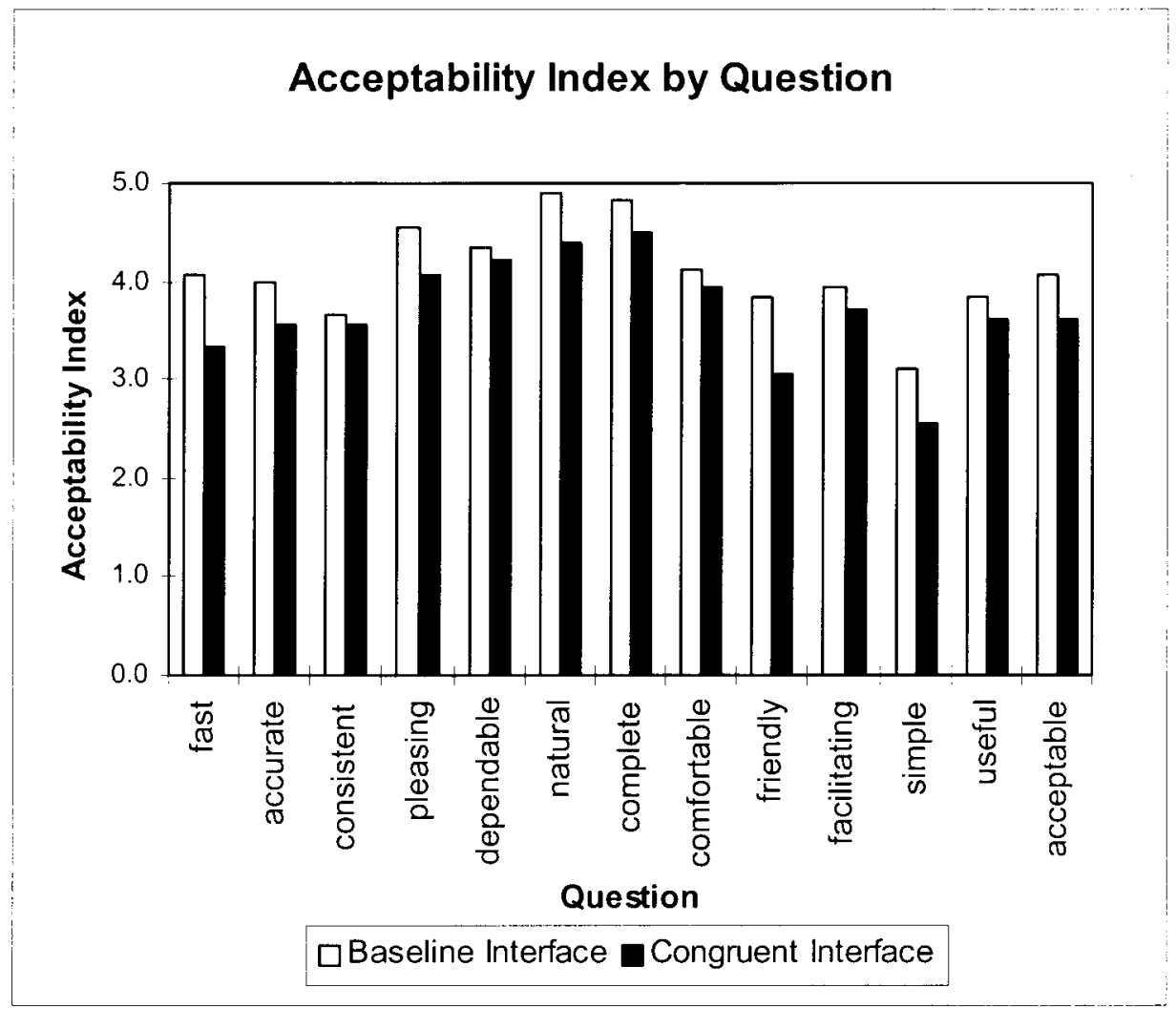

Fig. 5. Comparison of acceptability index by question (a lower value indictes greater user acceptance).

For analyzing the subjective scores, an acceptability index by question was defined as the mean scale response for each question across all participants. A lower AI was indicative of higher user acceptance. One subject's score was more than two standard deviations outside the mean AI and was rejected as an outlier. This person answered every question with the value of 1 , resulting in a mean AI of 1 . No other subject answered every question with the same value, suggesting that this person did not give ample consideration. With this outlier removed, the baseline interface AI was 3.99 , and the congruent interface was 3.63 , which was a modest $9.0 \%$ improvement. The result was significant using the $2 \times 13$ ANOVA $(\mathrm{p}=$ $0.014)$, and the interaction between groups was not $(\mathrm{p}=0.999)$. A comparison of these values is shown in Figure 5.

\section{DISCUSSION}

The results of this study showed that the congruent interface was favored over the baseline interface. This supported the hypothesis that the percep- 
tual structure of an input task is an important consideration when designing a multimodal computer interface. As shown in Table VI, the QM relationship compared the entry of integral attributes with an integral device in the congruent interface and a separable device in the baseline interface. Based on this, the three null hypotheses were rejected in favor of alternate hypotheses stating that performance, accuracy, and user acceptance were shown to improve when integral attributes are entered with a single device. However, since separable attributes were not tested with both integral and separable devices, no conclusion can be made about whether it was advantageous to enter separable attributes with either a single device or multiple devices.

With respect to accuracy, the results were only significant for speech errors. Mouse errors showed a slight improvement with the baseline interface, but these were not significant. This was possibly because there were few such errors recorded. Across all subjects, there were only 16 mouse errors compared to 175 speech errors. A mouse error was recorded when a subject clicked on the wrong item from a list and later changed his or her mind, which was rare.

Diagnosis errors showed a slight improvement with the congruent interface. There were 77 diagnosis errors, but the results were not statistically significant. Diagnosis errors were really a measure of the subject's expertise in identifying tissue types and reactions. Ordinarily, this type of finding would suggest that there is no relationship between perceptual structure of the input task and the ability of the user to apply domain expertise. However, this cannot be concluded, since efforts were made to avoid measuring one's ability to apply domain expertise by allowing subjects to review the tissue slides before the actual test.

As stated earlier, 175 speech errors were recorded during the 240 data entry tasks. Because some participants had to repeat a phrase more than once when there was a recognition error, there were altogether 415 commands uttered to complete the 240 data entry tasks. The actual accuracy rate on the command level was therefore 58\%. This was probably the most practical measure of accuracy, since the only way to correct an error was to repeat the entire command.

Speech accuracy was also measured at the word level, giving the probability that any single word spoken would be recognized correctly. Each of the 415 utterances consisted of an average of four words, so there were 175 speech errors over roughly 1,660 words. The accuracy rate at the word level was therefore about $89.5 \%$. This rate corresponds to the expected wordlevel accuracy rate of most continuous recognition dictation products of $87 \%$ to $95 \%$ [Alwang and Stinson 1998]. However, this may not be a practical measurement in this context.

A general understanding with speech recognition is that phrases with less syllables or that sound alike will have higher error rates. In the experiment, speech was used for entering the site and qualifier (SQ) in the baseline interface and the qualifier and morphology (QM) in the congruent interface. The average QM phrase was about $20 \%$ longer than the average 
SQ phrase (9.8 syllables versus 8.2 syllables). However, both phrases were reasonably long, and all of the sites and morphologies had unique pronunciations. Based on user training before the experiment, the greatest source of recognition errors was from qualifiers. This was most likely because the qualifiers consisted of shorter terms, many of which sounded alike. Since qualifiers were part of both the SQ and QM phrases, it was concluded that differences between sites and morphologies did not contribute significantly to the error rate.

Pearson correlation coefficients were computed to reveal possible relationships between the dependent variables. This included relationships between the baseline and congruent interface, relationships with task completion time, and relationships with user acceptance.

A positive correlation on time between the baseline interface and congruent interface was probably because a subject who works slowly (or quickly) will do so regardless of the interface $(p<0.001)$. The positive correlation of diagnosis errors between the baseline and congruent interface suggests that a subject's ability to apply domain knowledge was not effected by the interface $(\mathrm{p}<0.001)$, since the slides were reviewed beforehand.

The lack of correlation for speech errors was notable. Under normal circumstances, one would expect there to be a positive correlation, implying that a subject who made errors with one interface was predisposed toward making errors with the other. Having no correlation agrees with the finding that the user was more likely to make speech errors with the baseline interface, because the interface did not match the perceptual structure of the input task.

When comparing time to other variables, we found several relationships. There was a positive correlation between the number of speech errors and task completion time $(\mathrm{p}<0.01)$. This was expected, since it took time to identify and correct those errors. There was also a positive correlation between time and the number of mouse errors. However, due to the relatively few mouse errors recorded, nothing was inferred from these results. No correlation was observed between task completion time and diagnosis errors, since the slides were reviewed before the test.

Several relationships were identified between the acceptability index and other variables. Note that for the acceptability index, a lower score corresponds to higher user acceptance. A significant positive correlation was observed between acceptability index and the number of speech errors ( $p$ $<0.01$ ). An unexpected result was that no correlation was observed between task completion time and the acceptability index. This suggests that accuracy is more critical than speed, with respect to whether a user will embrace the computer interface. No correlation was found between the acceptability index and mouse errors, most likely due to the lack of recorded mouse errors. A significant positive correlation was observed between the acceptability index and diagnosis errors $(\mathrm{p}<0.01)$. The number of diagnosis errors was assumed to be inversely proportional to the domain expertise of each subject. What this finding suggests is that the 
more domain expertise a person has, the more he or she is likely to approve of the computer interface.

\section{SUMMARY}

A research hypothesis was proposed for multimodal speech and directmanipulation interfaces. It stated that multimodal, multidimensional interfaces work best when the input attributes are perceived as separable, and that unimodal, multidimensional interfaces work best when the inputs are perceived as integral. This was based on previous research that extended the theory of perceptual structure [Garner 1974] to show that performance of multidimensional, unimodal, graphical environments improves when the structure of the perceptual space matches the control space of the input device [Jacob et al. 1994]. Also influencing this study was the finding that contrastive functionality can drive a user's preference of input devices in multimodal interfaces [Oviatt and Olsen 1994] and the framework for complementary behavior between speech and direct manipulation [Cohen 1992].

A biomedical software prototype was developed with two interfaces to test this hypothesis. The first was a baseline interface that used speech and mouse input in a way that did not match the perceptual structure of the attributes, while the congruent interface used speech and mouse input in a way that best matched the perceptual structure. The results of this experiment supported the hypothesis that the perceptual structure of an input task is an important consideration when designing a multimodal computer interface. Task completion time, accuracy, and user acceptance all increased when a single modality was used to enter attributes that were integral. It should be noted that this experiment did not determine whether a unimodal speech-only or mouse-only interface would perform better overall. It also did not show whether separable attributes should be entered with separate input devices or one device.

A group of 20 clinical and veterinary pathologists evaluated the interface in an experimental setting, where data on task completion time, speech errors, mouse errors, diagnosis errors, and user acceptance were collected. Task completion time improved by $22.5 \%$; speech errors were reduced by $36 \%$; and user acceptance increased $9.0 \%$ for the interface that best matched the perceptual structure of the attributes. Mouse errors decreased slightly, and diagnosis errors increased slightly for the baseline interface; but these were not statistically significant. User acceptance was related to speech recognition errors and domain errors, but not task completion time.

Additional research into theoretical models which can predict the success of speech input in multimodal environments are needed. This could include a more direct evaluation of perceptual structure on separable data. Another approach could include studies on minimizing speech errors. The reduction of speech errors has typically been viewed as a technical problem of the speech recognition engine. However, this effort successfully reduced the rate of speech errors by applying certain user interface principles based on 
perceptual structure. Others have reported a reduction in speech errors by applying different user interface techniques [Oviatt 1996]. In addition, noting the strong relationship between user acceptance and domain expertise, additional research on how to build domain knowledge into the user interface might be helpful.

\section{ACKNOWLEDGMENTS}

The authors thank Judy Fetters and Alan Warbritton from the National Center for Toxicological Research for providing tissue slides and other assistance with the software prototype. The authors also thank Lowell Groninger, Greg Trafton, and Clare Grasso for help with the experiment design, and Tulay Adali, Charles K. Nicholas, and Anthony W. Norcio for serving as doctoral dissertation committee members at the University of Maryland Baltimore County. Finally, the authors thank those who graciously participated in this study from the University of Maryland Medical Center, the Baltimore Veteran Affairs Medical Center, the Johns Hopkins Medical Institutions, and the Food and Drug Administration.

\section{REFERENCES}

Alwang, G. And Stinson, C. 1998. Speech recognition: Finding its voice. PC Mag. 17, 18, 191-204.

Buxton, B. 1993. HCI and the inadequacies of direct manipulation systems. SIGCHI Bull. 25, 1 (Jan. 1993), 21-22.

CAsali, S. P., Williges, B. H., AND Dryden, R. D. 1990. Effects of recognition accuracy and vocabulary size of a speech recognition system on task performance and user acceptance. Hum. Factors 32, 2 (Apr. 1990), 183-196.

Cohen, P. R. 1992. The role of natural language in a multimodal interface. In Proceedings of the 5th Annual ACM Symposium on User Interface Software and Technology (UIST '92, Monterey, CA, Nov. 15-18), J. Mackinlay and M. Green, Eds. ACM Press, New York, NY, 143-149.

Cohen, P. R. And Oviatt, S. L. 1994. The role of voice in human-machine communication. In Voice Communication between Humans and Machines, D. B. Roe and J. G. Wilpon, Eds. National Academy Press, Washington, DC, 34-75.

Cole, R., Hirschman, L., Atlas, L., Beckman, M., Biermann, A., Bush, M., Clements, M., Cohen, J., Garcia, O., Hanson, B., Hermansky, H., Levinson, S., McKeown, K., Morgan, N., Novick, D. G., Ostendorf, M., Oviatt, S., Price, P., Silverman, H., Spitz, J., Waibel, A., Weinstein, C., Zahorian, S., AND Zue, V. 1995. The challenge of spoken language systems: Research directions for the Nineties. IEEE Trans. Speech Audio Process. 3, 1 (Jan.), 1-21.

Dillon, T. W., McDowell, D., Norcio, A. F., And DeHaemer, M. J. 1995. Nursing acceptance of a speech-input interface: A preliminary investigation. Comput. Nursing 12, 6, 264-271.

GARner, W. R. 1974. The Processing of Information and Structure. Lawrence Erlbaum Assoc. Inc., Hillsdale, NJ.

Graso, M. A. 1995. Automated speech recognition in medical applications. M.D. Comput. 12, $1,16-23$.

Grasso, M. A. And Grasso, C. T. 1994. Feasibility study of voice-driven data collection in animal drug toxicology studies. Comput. Bio. Med. 24, 4, 289-294.

Grasso, M. A., Ebert, D. S., AND Finin, T. W. 1997. Acceptance of a speech interface for biomedical data collection. In Proceedings of the AMIA 1997 Fall Symposium. American Medical Informatics Assoc., Washington, DC, 739-743.

Jacob, R. J. K., Sibert, L. E., McFarlane, D. C., And Mullen, M. P. 1994. Integrality and separability of input devices. ACM Trans. Comput. Hum. Interact. 1, 1 (Mar. 1994), 3-26. 
Jones, D. M., HAPEShi, K., AND FRAnkish, C. 1990. Design guidelines for speech recognition interfaces. Appl. Ergonom. 20, 1, 40-52.

Landau, J. A., Normich, K. H., And Evans, S. J. 1989. Automatic speech recognition-Can it improve the man-machine interface in medical expert systems?. Int. J. Biomed. Comput. 24, $111-117$.

NCTR. 1985. Post experiment information system pathology code table reference manual. TDMS Doc. 1118-PCT-4.0. National Center for Toxicological Research, Jefferson, AK.

Oviatt, S. L. 1996. Multimodal interfaces for dynamic interactive maps. In Proceedings of the ACM Conference on Human Factors in Computing Systems (CHI '96). ACM Press, New York, NY, 95-102.

Oviatt, S. L. AND Olsen, E. 1994. Integration themes in multimodal human-computer interaction. In Proceedings of the International Conference on Spoken Language Processing. Accoustical Society of Japan, Japan, 551-554.

PeAcocke, R. D. AND GRAF, D. H. 1990. An introduction to speech and speaker recognition. Computer 23, 8 (Aug. 1990), 26-33.

Pomerantz, J. R. And Lockhead, G. R. 1991. Perception of structure: An overview. In The Perception of Structure. American Psychological Association, Washington, DC, 1-20.

SHEPARD, R. N. 1991. Integrality versus separability of stimulus dimension: From an early convergence to evidence to a proposed theoretical basis. In The Perception of Structure. American Psychological Association, Washington, DC, 53-71.

ShNEIDERMAN, B. 1983. Direct manipulation: A step beyond programming languages. Computer 16, 8, 57-69.

ShNEIDERMAN, B. 1993. Sparks of Innovation in Human-Computer Interaction. Ablex Publishing Corp., Norwood, NJ.

Received: April 1997; revised: December 1997, September 1998, October 1998, and December 1998; accepted: December 1998 